\title{
Application of modified REFIT code for J-PARC/MLF to evaluation of neutron capture cross section on ${ }^{155,157} \mathrm{Gd}$
}

\author{
Kazuhito Mizuyama $^{1, a}$, Nobuyuki Iwamoto ${ }^{1}$, Osamu Iwamoto $^{1}$, Hiroyuki Hasemi ${ }^{2}$, Koichi Kino ${ }^{3}$, Atsushi Kimura ${ }^{1}$, \\ and Yoshiaki Kiyanagi ${ }^{4}$ \\ 1 Japan Atomic Energy Agency, Nuclear Science and Engineering Center, 2-4 Shirakata, Tokai-mura, Naka-gun, Ibaraki-ken 319-1195, \\ Japan \\ 2 Hokkaido University, Graduate School of Engineering, Kita 13 Nishi 8, Kita-ku, Sapporo 060-8628, Japan \\ 3 National Institute of Advanced Industrial Science and Technology, 1-1-1 Umezono, Tsukuba, Ibaraki 305-8568, Japan \\ ${ }^{4}$ Nagoya University, Graduate School of Engineering, 1 Furo-cho, Chikusa-ku, Nagoya 464-8603, Japan
}

\begin{abstract}
In order to analyze the experimental data measured by the Accurate Neutron-Nucleus Reaction Measurement Instrument (ANNRI) at the Japan Proton Accelerator Research Complex/Materials and Life Science Experimental Facility (J-PARC/MLF), it is necessary to take into account the double-bunch structure of the neutron pulse and the energy resolution function for the operational condition of the J-PARC/MLF. The modified REFIT code has been developed to treat the double-bunch neutron pulse and the energy resolution function for J-PARC/MLF. In this study, we applied the modified REFIT code to analyze the new data of the neutron capture cross section of ${ }^{155} \mathrm{Gd}$ and ${ }^{157} \mathrm{Gd}$ recently measured by ANNRI in the J-PARC/MLF, and obtained the resonance parameters of two Gd isotopes. We discussed the differences between the our obtained results and the other libraries.
\end{abstract}

\section{Introduction}

There are mainly two difficulties for the evaluation of the data in neutron time-of-flight (TOF) measurements by the Accurate Neutron-Nucleus Reaction Measurement Instrument (ANNRI) at the Japan Proton Accelerator Research Complex/Materials and Life Science Experimental Facility (J-PARC/MLF), i.e., the double-bunch structure and the resolution function of the pulsed neutron beam. The former is caused by an incident proton delivered by a doublebunch scheme. This makes the structure of resonance split into two peaks. The latter gives the energy spread of pulsed neutron mainly from source and moderator. This affects the resonance shape.

The least-squares multilevel R-matrix code REFIT $[1,2]$ has been widely used for the resonance analysis. In order to overcome the difficulties mentioned above, REFIT was modified to include the function of describing the double bunch structure and the resolution function for the ANNRI [3]. The parameters of resolution function based on the formulation of Ikeda-Carpenter [4] were obtained at certain operational conditions of J-PARC/MLF [5]. Nevertheless, this code has not been applied to the actual evaluation of nuclear data.

Gadolinium has been used as neutron-absorbing material in a thermal reactor since ${ }^{155,157} \mathrm{Gd}$ have large thermal neutron capture cross sections. However, there is a discrepancy between the previously measured data and JENDL-4.0 [6] data for ${ }^{157} \mathrm{Gd}$. For example, the data measured at the RPI [7] make the criticality of Gd-loaded thermal systems in ICSBEP overestimated [8].

\footnotetext{
a e-mail: mizuyama.kazuhito@jaea.go.jp
}

Recently, the neutron capture cross sections of ${ }^{155} \mathrm{Gd}$ and ${ }^{157} \mathrm{Gd}$ were measured by the TOF method with an $\mathrm{NaI}(\mathrm{TI})$ spectrometer of ANNRI in the J-PARC/MLF using the Gadlinium nitrate $\left(\mathrm{Gd}\left(\mathrm{NO}_{3}\right)_{3}\right)$ sample. The absolute values of the cross sections for lower energy region $\left(<0.1 \mathrm{eV}\right.$ for ${ }^{155} \mathrm{Gd}, 0.2 \mathrm{eV}$ for $\left.{ }^{157} \mathrm{Gd}\right)$ were obtained with good accuracy by a new method invented by Kimura et al. [9]. By taking ratio of capture yields for thick and thin samples, the neutron flux and the detector efficiency are canceled out in a new method. Under the condition $\exp \left(-\sigma\left(E_{n}\right) N_{\text {thick }}\right) \sim 0$, the absolute cross section can be obtained from the ratio of capture yield with accuracy, where $\sigma\left(E_{n}\right)$ is the capture cross section at neutron energy $E_{n}$ and $N_{\text {thick }}$ is the thickness of the thick sample.

In this study, we apply the modified REFIT code to the analysis of the capture cross sections of ${ }^{155,157} \mathrm{Gd}$, and demonstrate the applicability of the code, and derive the resonance parameters for some low-lying resonances of the two Gd isotopes, and discuss the difference between the new resonance parameters and other libraries.

\section{Resonance analysis}

\subsection{Sample information}

In this section, we introduce the experimental information used for the resonance analysis by the modified REFIT.

The neutron capture cross section of ${ }^{155} \mathrm{Gd}$ and ${ }^{157} \mathrm{Gd}$ were measured by using the Gadlinium nitrate $\left(\mathrm{Gd}\left(\mathrm{NO}_{3}\right)_{3}\right)$ sample. The isotopic enrichment of ${ }^{155} \mathrm{Gd}$ sample was $91.7 \% ;{ }^{156} \mathrm{Gd}$ and ${ }^{157} \mathrm{Gd}$ were contained by $5.1 \%$ and $1.1 \%$ respectively. The isotopic enrichment of ${ }^{157} \mathrm{Gd}$ sample was 


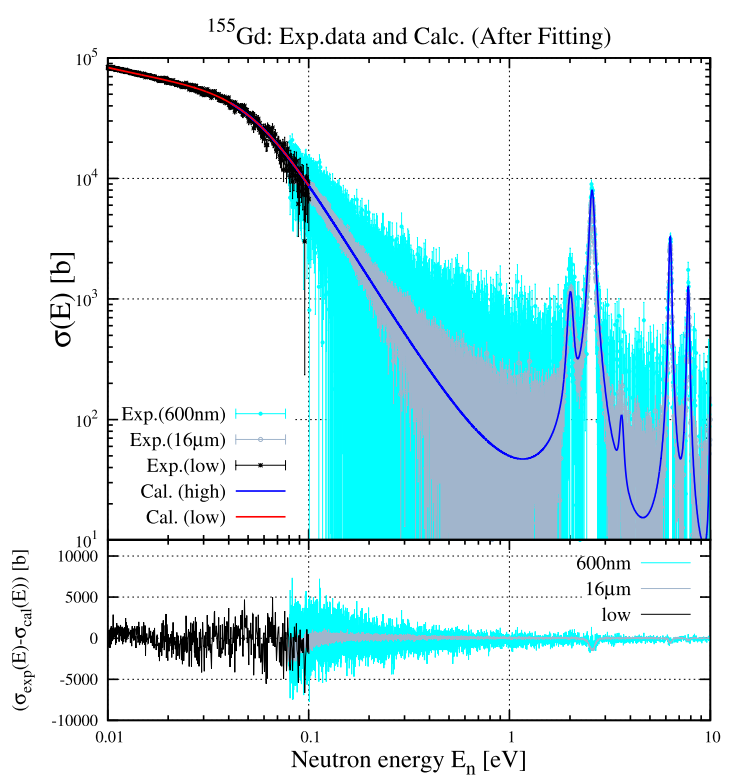

Figure 1. An overview of the neutron capture cross section data measured by ANNRI in J-PARC/MLF for ${ }^{155} \mathrm{Gd}$ within the energy range $0.01 \mathrm{eV}<E_{n}<10 \mathrm{eV}$ and the fitting results by the modified REFIT are shown in the upper panel. The difference between the fitted result and the experimental data is shown in the lower panel.

$88.3 \%$; $9.1 \%$ of ${ }^{158} \mathrm{Gd}, 1.6 \%$ of ${ }^{156} \mathrm{Gd}$ and $0.3 \%$ of ${ }^{155} \mathrm{Gd}$ were contained as impurities.

For higher energy region $\left(>0.1 \mathrm{eV}\right.$ for ${ }^{155} \mathrm{Gd}, 0.2 \mathrm{eV}$ for ${ }^{157} \mathrm{Gd}$ ), the dead-time correction, the background subtraction, and the self-shielding \& multiple scattering correction were carried out in order to obtain the relative cross section. The measured relative cross sections of ${ }^{155} \mathrm{Gd}$ with $16 \mu \mathrm{m}$ and $600 \mathrm{~nm}$-thick samples were normalized at $0.1 \mathrm{eV}$ to the absolute cross section in lower energy region by concerning the available condition of a new method for lower energy data and the uncertainty of the correction of the self-shielding effect as shown in Fig. 1. The relative cross sections of ${ }^{157} \mathrm{Gd}$ with $20 \mu \mathrm{m}$ and $600 \mathrm{~nm}$-thick samples were normalized at $0.2 \mathrm{eV}$ as shown in Fig. 2.

\subsection{Analysis by modified REFIT}

In this study, the resonance parameters, neutron width $\Gamma_{n}$, radiation width $\Gamma_{\gamma}$, and resonance energy $E_{0}$ of ${ }^{155,157} \mathrm{Gd}$ below $10 \mathrm{eV}$ were determined from the TOF measurements in the J-PARC/MLF/ANNRI by using the modified REFIT code. The energy resolution parameter for the ANNRI derived by Kino et al. [5] was adopted in order to take into account the effect of the double-bunch proton beam. The effective temperature was $300 \mathrm{~K}$. The TOF channel width of $1 \mu \mathrm{s}$ and the flight length of $27.98 \mathrm{~m}$ were used as input parameters. The abundance of impurities of samples was taken into account for the calculation. The same spin assignments as JENDL-4.0 were assumed for analysed resonances. The data in both lower and higher energy regions below $10 \mathrm{eV}$ were fitted simultaneously by the modified REFIT code.

Fitting results are shown in Fig. 1 and Fig. 2. Both data of ${ }^{155} \mathrm{Gd}$ and ${ }^{157} \mathrm{Gd}$ were fitted reasonably well to the experimental data. It should be noted that a negative resonance which does not exist in JENDL-4.0 originaly

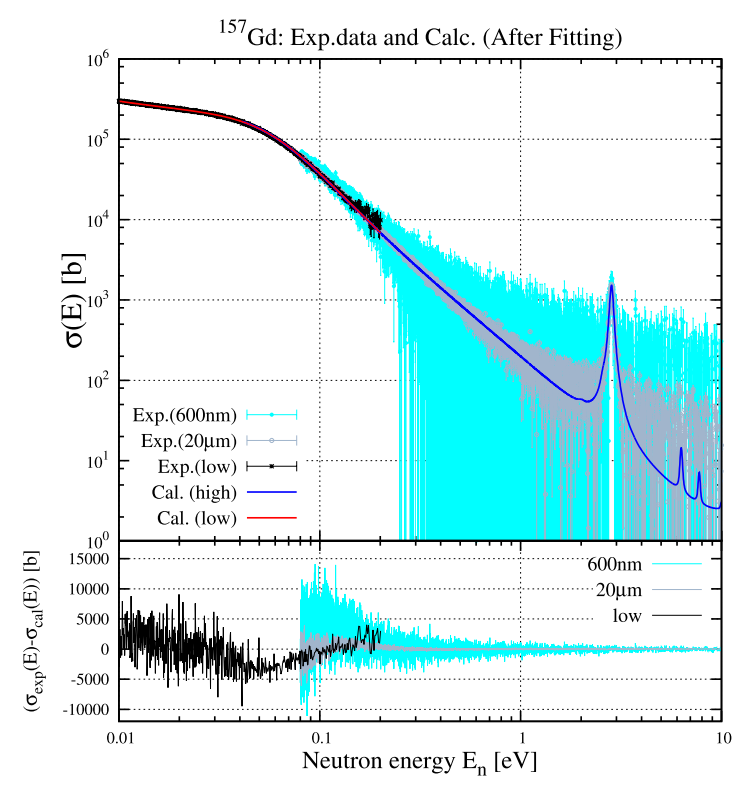

Figure 2. Same figure as Fig. 1 but for ${ }^{157} \mathrm{Gd}$.

Table 1. Resonance parameters of ${ }^{155} \mathrm{Gd}$ up to $3 \mathrm{rd}$ resonance and comparisons with the other libraries.

\begin{tabular}{ccccc}
\hline \hline Data & $\begin{array}{c}E_{0} \\
{[\mathrm{eV}]}\end{array}$ & $\mathrm{J}$ & $\begin{array}{c}\Gamma_{n} \\
{[\mathrm{meV}]}\end{array}$ & $\begin{array}{c}\Gamma_{\gamma} \\
{[\mathrm{meV}]}\end{array}$ \\
\hline Present & 0.0266 & 2 & 0.0908 & 94.28 \\
& 2.0141 & 1 & 0.2715 & 97.09 \\
JENDL-4.0 [6] & 2.5793 & 2 & 1.9012 & 111.3 \\
& 0.0251 & 2 & 0.0970 & 104.0 \\
& 2.0120 & 1 & 0.4000 & 128.0 \\
JEFF-3.2 [10] & 2.5729 & 2 & 1.7060 & 107.1 \\
& 0.0268 & 2 & 0.1040 & 108.0 \\
ENDF/B-VII.1 [11] & 2.0080 & 1 & 0.3707 & 110.0 \\
& 2.5680 & 1 & 2.8933 & 111.0 \\
& 0.0268 & 2 & 0.1040 & 108.0 \\
& 2.0080 & 1 & 0.3707 & 110.0 \\
\hline \hline & 2.5680 & 2 & 1.7440 & 111.0 \\
\hline
\end{tabular}

Table 2. Resonance parameters of ${ }^{157} \mathrm{Gd}$ up to 2 nd resonance.

\begin{tabular}{ccccc}
\hline \hline Data & $\begin{array}{c}E_{0} \\
{[\mathrm{eV}]}\end{array}$ & $\mathrm{J}$ & $\begin{array}{c}\Gamma_{n} \\
{[\mathrm{meV}]}\end{array}$ & $\begin{array}{c}\Gamma_{\gamma} \\
{[\mathrm{meV}]}\end{array}$ \\
\hline Present & -0.4069 & 1 & 3.0368 & 216.4 \\
& 0.0312 & 2 & 0.3953 & 94.13 \\
JENDL-4.0 & 2.8366 & 2 & 0.4046 & 134.9 \\
& 0.0320 & 2 & 0.4280 & 107.0 \\
JEFF-3.2 & 2.8287 & 2 & 0.3770 & 109.7 \\
& 0.0314 & 1 & 0.7867 & 106.0 \\
ENDF/B-VII.1 & 2.8250 & 2 & 0.3448 & 97.0 \\
& 0.0314 & 2 & 0.4740 & 107.2 \\
& 2.8250 & 2 & 0.3448 & 97.00 \\
\hline \hline
\end{tabular}

was necessary to reproduce the slope shape between the 1 st and 2 nd resonances of ${ }^{157} \mathrm{Gd}$. The obtained low-lying resonance parameters of ${ }^{155} \mathrm{Gd}$ and ${ }^{157} \mathrm{Gd}$ are shown in Table 1 and Table 2, respectively. Resonance parameters of JENDL-4.0 [6], JEFF-3.2 [10] and ENDF/B-VII.1 [11] are listed together for comparison. The neutron capture cross section for ${ }^{155} \mathrm{Gd}$ and ${ }^{157} \mathrm{Gd}$ calculated by obtained resonance parameters are shown in Fig. 3 and Fig. 4 respectively. The thermal region of those cross sections are shown in Fig. 5 and Fig. 6, the 2nd (or 3rd) resonance 


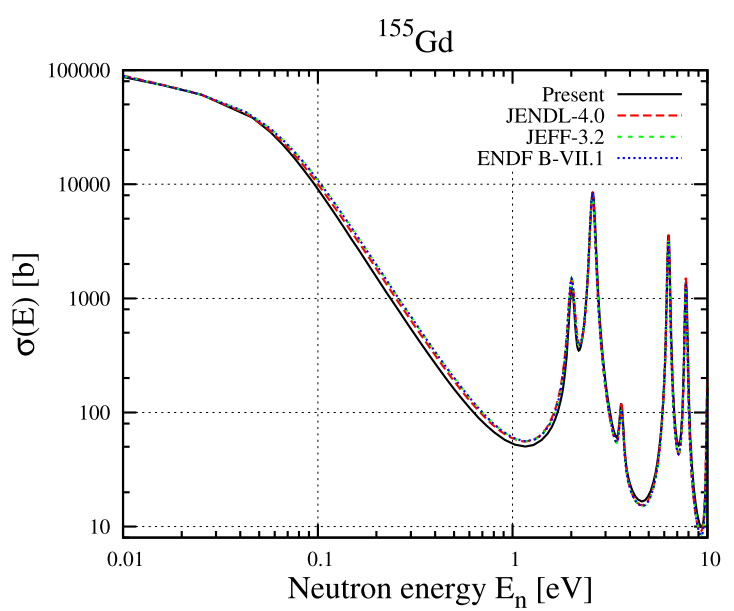

Figure 3. Overview of the neutron capture cross section of ${ }^{155} \mathrm{Gd}$ calculated with the resulting resonance parameters from the analysis of the experimental data measured by ANNRI in J-PARC/MLF.

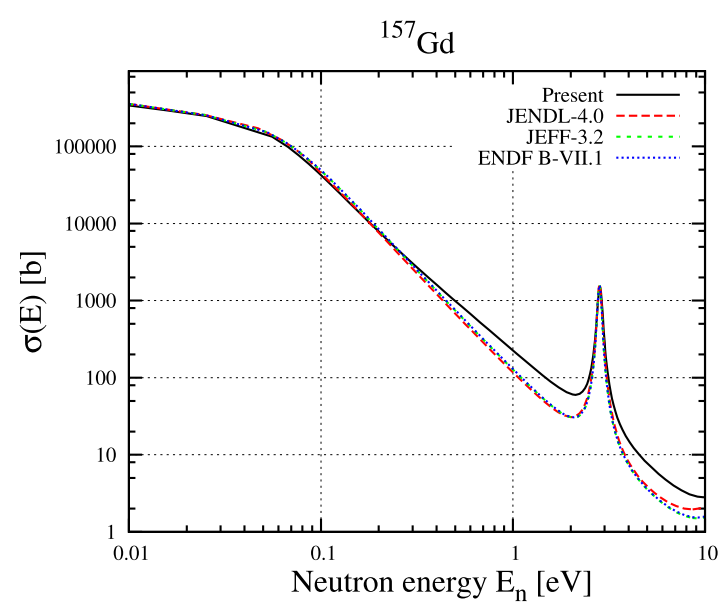

Figure 4. Same figure as Fig. 3 but for ${ }^{157} \mathrm{Gd}$.

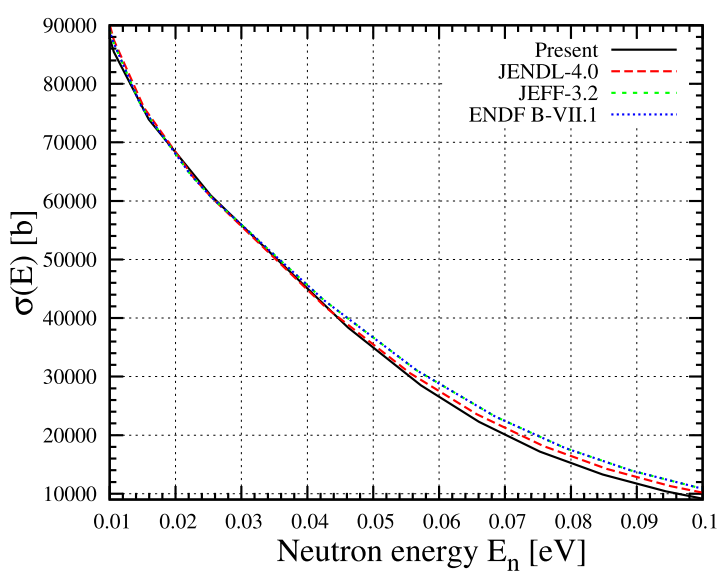

Figure 5. The calculated neutron capture cross section of ${ }^{155} \mathrm{Gd}$ for the thermal energy region.

region of cross section are shown in Fig. 7 and Fig. 8. The figures include the calculated cross sections by the JENDL-4.0, JEFF-3.2 and ENDF/B-VII.1 for comparison.

There is not so large difference of thermal neutron capture cross section of ${ }^{155} \mathrm{Gd}$ between our result and other libraries as shown in Fig. 5, but in the energy range of $0.1 \mathrm{eV}<E_{n}<1.0 \mathrm{eV}$, the resulting cross section is smaller than the other libraries. This is due to the fact that

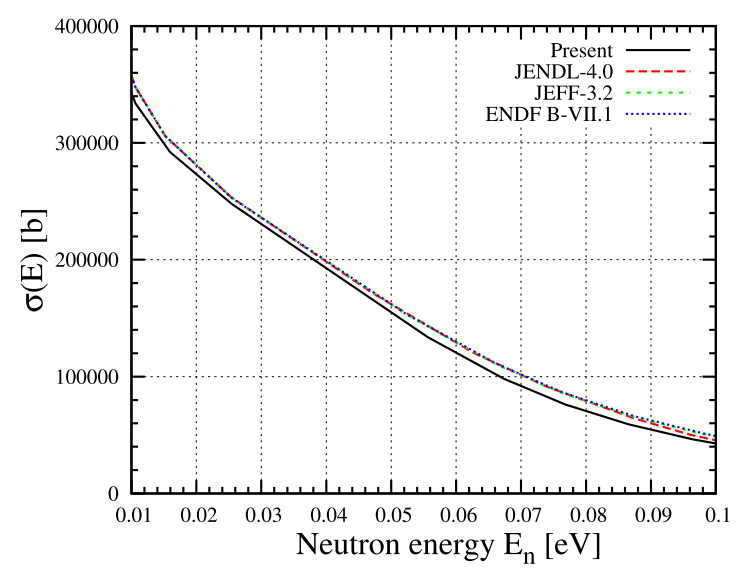

Figure 6. Same figure as Fig. 5 but for ${ }^{157} \mathrm{Gd}$.

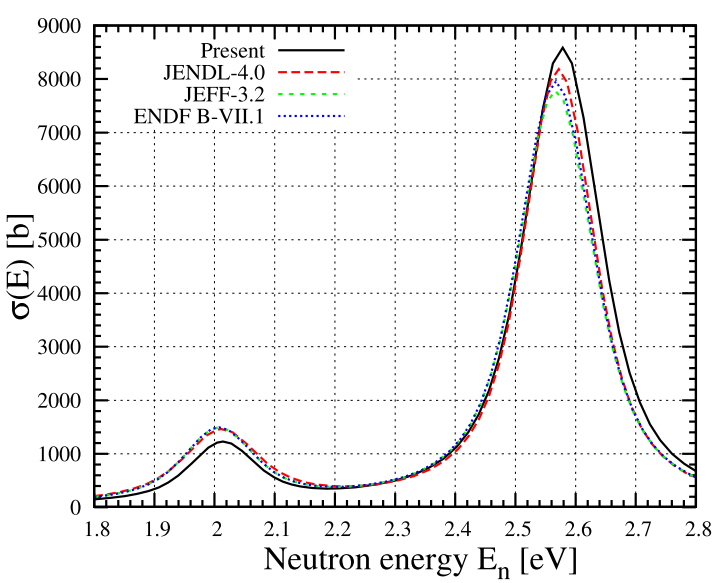

Figure 7. The calculated capture cross section of ${ }^{155} \mathrm{Gd}$ for the region of 2 nd and 3 rd resonances.

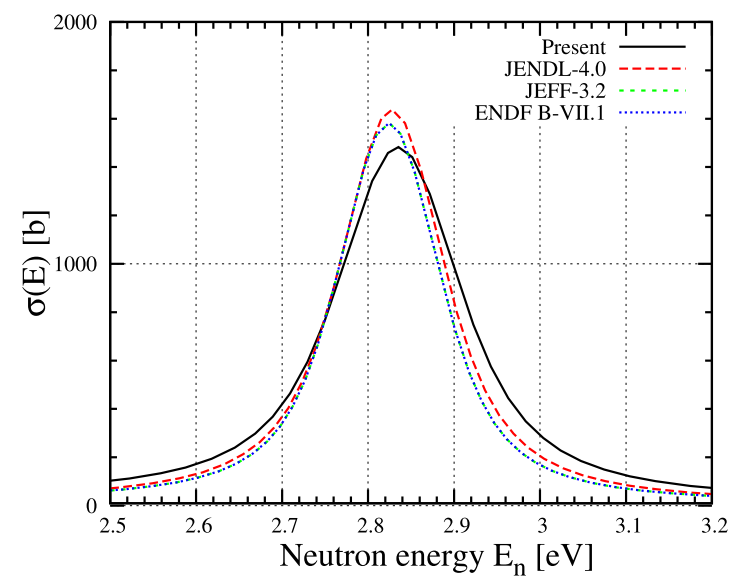

Figure 8. Same figure as Fig. 7 but for ${ }^{157} \mathrm{Gd}$.

the gamma width of 1 st and 2 nd resonances of ${ }^{155} \mathrm{Gd}$ are smaller than the other libraries. In the case of ${ }^{157} \mathrm{Gd}$, our obtained thermal cross section is slightly smaller than other libraries in the thermal energy region and is larger than the other libraries in the energy range of $0.1 \mathrm{eV}<E_{n}<$ $1.0 \mathrm{eV}$.

The accuracy of the absolute values of the lower energy below $0.1 \mathrm{eV}$ is reliable in principle of the new method. However, the relative cross section data measured for the higher energy region above $0.1 \mathrm{eV}$ were sensitive to the correction for the self-shielding effect around normalized point of energy. Therefore, the more careful investigation 
by performing the measurements using thinner sample to reduce the uncertainty of the self-shielding correction would be necessary to confirm our results of this study. The simultaneous analysis of the data of neutron capture and transmission is also important to justify our obtained resonance parameters.

\section{Summary}

We analyzed new neutron capture cross section data of ${ }^{155,157} \mathrm{Gd}$ by using a modified REFIT code to take into account the energy resolution function and the doublebunch neutron pulse shape of J-PARC/MLF. It was found that the cross section data of ${ }^{157} \mathrm{Gd}$ could be fitted reasonably well by introducing a negative resonance, but the fitted cross section in the energy range between the 1 st and 2 nd resonances was larger than the other libraries. The more careful investigation would be necessary to confirm our results of analysis.

The authors are grateful for the financial support of "Research and development for Accuracy Improvement of neutron nuclear data on Minor ACtinides (AIMAC)" project entrusted to Japan Atomic Energy Agency by Ministry of Education, Culture, Sport, Science and Technology of Japan (MEXT).

\section{References}

[1] M.C. Moxon, J.B. Brisland, GEEL REFIT, A least squares fitting program for resonance analysis of neutron transmission and capture data computer code, AEA-InTec -0630, AEA Technology, October (2008)

[2] P. Schillebeeckx et al., Nucl. Data Sheets 113, 3054 (2012)

[3] H. Hasemi et al., Nucl. Instrum. Methods A 773, 137 (2015)

[4] S. Ikeda, J.M. Carpenter, Nucl. Instrum. Methods A 239, 536 (1985)

[5] K. Kino et al., Nucl. Instrum. Methods Phys. Res. Sect. A 736, 66 (2014)

[6] K. Shibata et al., J. Nucl. Sci. Technol. 48(1), 1-30 (2011)

[7] G. Leinweber et al., Nucl. Sci. Eng. 154, 261-279 (2000)

[8] G. Chiba et al., J. Nucl. Sci. Technol. 48, 172-187 (2011)

[9] A. Kimura et al., in preparation

[10] https://www . oecd-nea.org/dbforms/data/ eva/evatapes/jeff_32/;2014

[11] M.B. Chadwick et al., Nuclear Data Sheets 107, 2931-3060 (2006) 